\title{
Comparative transductions of breast cancer cells by three DNA viruses
}

\author{
Annick Lucas, Eric J. Kremer ${ }^{+}$, Silvio Hemmi *, José Luis \#, Françoise Vignon and \\ Gwendal Lazennec ${ }^{\text {II }}$
}

INSERM U540 "Molecular and Cellular Endocrinology of Cancers",

60, rue de Navacelles - 34090 Montpellier, France

+ Institut de Génétique Moléculaire de Montpellier, CNRS-UMR 5535,

1919 Route de Mende, 34293 Montpellier, France

* Molecular Biology I, University of Zurich,

Winterthurerstr. 190, Building 55L, 8057 Zurich, Switzerland

\# Laboratoire de Biochimie Cellulaire, CNRS-UMR 6032, Faculte de Pharmacie

27, bd Jean Moulin, 13385 Marseille Cedex 05, France

Running title: Breast cancer cell virus infection

\section{${ }^{\text {II }}$ Corresponding Author:}

Dr Gwendal Lazennec

INSERM U540 " Molecular and Cellular Endocrinology of Cancers ", 60, rue de Navacelles - 34090 Montpellier, France

Tel: (33) 4670430 84; Fax: (33) 467543084

E-mail: lazennec@montp.inserm.fr 


\begin{abstract}
Defining the ideal vectors to transduce breast cancer using viruses is currently under intense pre-clinical evaluation. Our study constitutes the first direct comparison of the infection efficiencies of a human serotype 5 (Ad5), a canine serotype 2 (CAV-2) adenovirus, and a human serotype 2 adeno-associated virus (AAV-2) in breast cancer cells. We observed an excellent infection efficiency for Ad5 vector, whereas both CAV-2 and AAV-2 vectors lead to low infection of these cells. Real-time PCR, flow cytometry and antibody blocking studies suggest that Ad5 and CAV-2 infection ability is not strictly dependent on coxsackie adenovirus receptor (CAR) or $\alpha_{v}$ integrin levels. In conclusion, our data suggest that human adenoviruses are excellent transducers of breast cancer cells, though it may be difficult to predict the extent of infection solely on CAR or $\alpha_{v}$ integrin levels.
\end{abstract}

Key words: breast, cancer, adenovirus, adeno-associated virus 


\section{Introduction}

Breast cancer is one of the leading causes of gynaecological cancer mortality in western countries. Approximately $10 \%$ of women will suffer from breast cancer within their life-span (1), where two thirds of tumors are estrogen-receptor (ER) positive. Endocrine therapy contributes significantly to prolonging the disease-free period post-surgery of only $50 \%$ of the patients (2), and therefore many investigators have proposed alternative therapies including gene transfer approaches. The cancer gene therapy strategies involve expression of growth inhibitors, tumor suppressors, pro-apoptotic, or tumor sensitizing genes (for review, (3)), and success critically depends on the number of initially transduced tumor cells.

Adenovirus (Ad) vectors are currently the most frequently used vectors for cancer gene therapy (4). They lead to a transient, robust, transgene expression, and efficient in vivo gene transfer has been reported in numerous tissues. The most recent Ad vectors, helper-dependent (high capacity, or gutless) are devoid of viral genes, have a cloning capacity of $28 \mathrm{~kb}$, and lead to expression for several months in vivo. However, greater than $80 \%$ of the adult population has detectable levels of circulating antibodies directed against antigens from human Ad, potentially reducing their efficiency (4). For these reasons, Ads derived from canine, bovine or avian species, for which there would be a low or non-existing seroprevalence in humans, have been evaluated for their potential in gene therapy (5). In addition, Adeno-associated virus (AAV) vectors have also generated great interest in gene therapy field because they efficiently transduce both dividing and quiescent cells (6) and display long-term expression in vivo. However, the use of AAV has encountered some limitations in its development such as production of high titers and generation of packaging cell lines (6).

The first critical step preceding any gene therapy strategy is to ensure that the vector transduces the target cells, which is the limiting factor for therapeutic effects in cancer gene therapy. Gene therapy for cancer offers novel possibilities that will eventually lead to the destruction of tumor cells. More 
than 3,500 patients have been enrolled in cancer gene therapy trials (4), where a majority of the approaches have used Ads. Most clinical trials have been small Phase I/II studies with the main objective of demonstrating safety and gene transfer (4). However, the choice of the ideal vector, which is able to transduce a large number of tumor types, remains to be identified. Data concerning the use of viruses to transduce breast cancer cells are still limited. The goal of this study was to evaluate the possible use of three promising types of vectors (Ad5, CAV-2 and AAV-2) in terms of their ability to transduce breast cancer cells.

\section{Materials and Methods}

\section{Cell culture}

Estrogen receptor positive (EFM-19, MCF-7, T47-D, ZR-75-1) or negative (MDA-MB-231, MDA-MB-435, MDA-MB-436, SKBR-3) breast cancer cell lines were obtained from ATCC. EFM-19 cells which were a gift of Dr. F. Hölzel (Hamburg, Germany) (7). Cells were routinely maintained in media recommended by ATCC supplemented with $10 \%$ foetal calf serum (FCS) and Gentamycin. For infection assays and FACS analysis, cells were seeded on 6-well plates.

\section{RNA extraction, reverse transcription and quantitative PCR}

Total RNA was isolated with TRIzol reagent (Invitrogen) as described by the manufacturer. Reverse transcription was performed using $5 \mu \mathrm{g}$ total RNA, random primers and Superscript II enzyme (Invitrogen). Quantitative PCR was performed with FastStart DNA Master SYBR Green I kit (Roche, Manheim, Germany) and 1:2000 of reverse transcription reaction on a Light Cycler instrument (Roche) as specified by the manufacturer. Ribosomal protein S9 (rS9) was used as an internal control. Primers (s: sense, as: anti-sense) used were: 
CARs: GCCTTCAGGTGCGAGATGTTAC

CARas: TCGCACCCATTCGACTTAGA

RS9s: AAGGCCGCCCGGGAACTGCTGAC

RS9as: ACCACCTGCTTGCGGACCCTGATA

\section{Vectors and infections}

The Ad5, CAV-2 and AAV-2 vectors expressing $\beta$-galactosidase (Ad $\beta$ gal, CAV $\beta$ gal and AAV $\beta$ gal) have been described previously (8-10). Ad $\beta$ gal stocks contained 1.4 x $10^{13}$ particles/ml, with a particle/infectious unit ratio of approximately 10 to 1 . CAV $\beta$ gal stocks contained $5.0 \times 10^{12}$ particles/ml, with a particle/infectious unit ratio of approximately 10 to 1 . AAV $\beta$ gal stocks contained $1.3 \times 10^{11}$ particles/ml, with a particle/infectious unit ratio of approximately 10 to 1. Cell lines were incubated overnight at different multiplicities of infection (MOI) (based on infectious units) in DMEM/F12 10\% CDFCS. The following day, the medium was replaced. The cells were fixed $48 \mathrm{~h}$ post-infection, and stained for $\beta$-galactosidase activity for $4 \mathrm{~h}$ at $37^{\circ} \mathrm{C}$ as previously described (10). Cells were then washed twice with PBS and photographed.

\section{FACS analysis}

To analyze CAR and integrin expression, cells were washed with PBS, detached by treatment with PBS/20 mM EDTA for 10 to $20 \mathrm{~min}$ at room temperature, resuspended in BSS/2\% FCS \{BSS: $0.14 \mathrm{M}$ $\left.\mathrm{NaCl}, 1 \mathrm{mM} \mathrm{CaCl}, 5.4 \mathrm{mM} \mathrm{KCl}, 0.8 \mathrm{mM} \mathrm{MgSO}_{4}, 0.3 \mathrm{mM} \mathrm{NaH}_{2} \mathrm{HPO}_{4}, 0.4 \mathrm{mM} \mathrm{KH}_{2} \mathrm{PO}_{4}(\mathrm{pH} 6.9)\right\}$, then collected by centrifugation. Approximately $10^{6}$ cells were used for specific staining for 30 minutes at $4^{\circ} \mathrm{C}$, using either $50 \mu \mathrm{l}$ of E1-1, a mouse anti-CAR antibody (11), $1 \mu \mathrm{g}$ of mouse anti- $\alpha_{\mathrm{v}} \beta_{5}$ antibody (MAB1961Z, Chemicon) or rabbit anti- $\alpha_{v}$ antibody (69.6.5) (12) in $250 \mu \mathrm{l}$ BSS/5\% FCS. 69.6 .5 recognises $\alpha v \beta 3, \alpha v \beta 5, \alpha v \beta 6$, and probably $\alpha v \beta 1$ and $\alpha v \beta 8$ integrins. If unlabelled primary antibodies were used, cells were rinsed in cold $\mathrm{BSS} / 2 \% \mathrm{FCS}$, followed by incubations with $1 \mu \mathrm{g}$ of phycoerytrin-labelled secondary conjugate (Beckman Coulter, Villepinte Roissy, France). The cells were washed prior to FACS analysis (Epics-XL flow cytometer (Beckman Coulter, Fullerton, CA). 


\section{Blocking studies}

E1-1 anti-CAR antibody $(500 \mu \mathrm{l})$ or $69.6 .5(10 \mu \mathrm{g} / \mathrm{ml})$ antibody were incubated with 2 X10 $0^{5}$ cells $(12$ well plates) for 20 min on ice. Ad $\beta$ gal or CAV $\beta$ gal viruses were then added to the medium and the incubation was continued for $1 \mathrm{~h}$ at $37^{\circ} \mathrm{C}$. Results were compared to infections carried out in the presence of whole mouse serum.

\section{Results}

\section{Infection assays}

In order to analyze the infection of breast cancer cell lines by Ad5, CAV-2 or AAV-2, we assayed a collection of 8 commonly used breast cancer cell lines. Increasing the MOI of Ad $\beta$ gal led to a notable increase in infection of most, but not all, cell lines (Fig. 1A). In particular, significant differences in terms of sensitivity were observed, allowing the separation of the cell lines in four relative groups: highly (MDA-MB-436 and SKBR-3), moderately (MDA-MB-231, MCF-7, T47-D, poorly (MDA-MB-435, ZR-75-1) and not transduced cells (EFM-19). The quantification of positive blue stained cells by microscopy (Fig.1B) further confirmed the inefficient infection of EFM-19 and the excellent transduction of MDA-MB-436 and SKBR-3 cells.

CAV-2 and AAV-2 vectors are currently under investigation for their clinical potential (8, 13), but so far, they have never been tested for their ability to transduce breast cancer cells. Results of infection with these two vectors are indicated in table 1 . We first observed that CAV $\beta$ gal was inefficient in infecting half of the cell lines tested. For cells transduced by CAV $\beta$ gal (MDA-MB-436, SKBR-3, MCF-7, T47-D), tropism was not exactly the same as for Ad $\beta$ gal virus. For instance, cells such as MDA-MB-231 which were moderately transduced by Adßgal, were poorly transduced by 
CAV $\beta$ gal. These data suggest that, at least in these cells, Ad5 used a receptor, that CAV-2 cannot. Surprisingly, AAV-2 vector was even less efficient than CAV-2 with the exception of MDA-MB-436 and SKBR-3 cells (Table 1). Our data indicate that AAV-2 is poorly suited for breast cancer gene therapies.

\section{Receptors: RNA and protein levels}

We next investigated the possible reasons underlying the tropism of Ad $\beta$ gal and CAV $\beta$ gal viruses towards breast cancer cell lines. The 46-kDa coxsackie and adenovirus receptor (CAR) (11) as well as $\alpha_{\mathrm{v}}$ integrins (14) are considered as the most relevant receptors of these viruses respectively. We first analyzed the mRNA levels of these two targets by quantitative PCR (Fig. 2). CAR expression was relatively variable among the different cell lines (Fig. 2A, left panel). Interestingly, CAR expression correlated poorly with the infectivity of Ad $\beta$ gal. For example, cells transduced inefficiently by Adßgal (EFM-19) showed the same level of CAR mRNA as moderately transduced cells such as MDA-MB-231, whereas readily transduced cells had moderate to high level of CAR. This holds also true for CAV $\beta$ gal for which cells with moderate levels of CAR (ZR-75-1) showed lower infection by CAV $\beta$ gal compared to cells expressing low levels of CAR (MCF-7). These data are in agreement with our current understanding of Ad5 and CAV-2 infection, suggesting that if CAR appears to be the primary cell surface protein used by virus for attachment, it may not be sufficient for maximal transduction.

For $\alpha$ v integrin mRNA levels, no obvious correlation with the infection efficiency was found (Fig. 2A, right panel). Cells such as SKBR-3 and EFM-19 showing low levels of $\alpha \mathrm{v}$ RNA could be highly (SKBR-3) or poorly transduced (EFM-19). On the contrary, cells expressing high levels of $\alpha \mathrm{v}$ integrins (MDA-MB-231 and MDA-MB-435) exhibited high and low transducing potentials respectively. 
Because protein and RNA levels occasionally do not match, we measured CAR, total $\alpha \mathrm{v}$, and $\alpha v \beta 5$ levels by flow cytometry using specific antibodies (Fig. 2B, C). In these human breast cancer cell lines, we found a good correlation between RNA and protein levels for CAR or $\alpha$ v. Not surprisingly, $\alpha v \beta 5$ integrins expression levels were not in agreement with the overall levels of $\alpha \mathrm{v}$ integrins ( $\alpha \mathrm{v}$ integrins can form heterodimers with at least 5 other $\beta$ chains). Moreover, $\alpha \mathrm{v} \beta 5$ integrins did not correlate better with the tropism of Ad $\beta$ gal and CAV $\beta$ gal viruses, suggesting that these integrins may contribute only modestly to the tropism of these viruses.

$\alpha v \beta 5$ integrin has also been reported as a co-receptor for AAV-2 (15), but MDA-MB-436 and SKBR-3 showed respectively low and high levels of this integrin, which suggests that this is not main determinant for the infection of these two types of cells.

\section{Blocking studies}

To confirm the implication of CAR and integrins in breast cancer cells infection by Ad $\beta$ gal and CAV $\beta$ gal, we evaluated this issue by competition studies performed in the presence of blocking antibodies. These experiments were performed on the three cell lines (MDA-MB-436, MCF-7 and T47-D) which were most efficiently transduced by both viruses. As seen in Fig. 3, incubation of $\alpha \mathrm{V}$ antibody did not affect significantly Ad $\beta$ gal and CAV $\beta$ gal infection in the three cell lines, confirming that integrins do not play a major role in virus infection. On the other hand, CAR antibody respectively decreased by $90 \%$ the infection of MDA-MB-436 cells by Ad $\beta$ gal and by about $60 \%$ the transduction of MCF-7 and T47-D cells. CAR antibody also decreased by $60 \%$ MDA-MB-436 infection by CAV $\beta$ gal but had no effect on MCF-7 and T47-D infection with this virus. T47-D cells show similar levels of CAR compared to MDA-MB-436 cells, but are less affected than MDA-MB-436 by CAR blockage, which suggests that Adßgal infection of MDA-MB-436 is primarily dependent on CAR, whereas the one of T47-D is most probably involving other surface 
proteins. So, it is likely that CAR levels measure could not be sufficient to predict Ad $\beta$ gal infection of breast cancer cells. Moreover, it is important to note that CAV $\beta$ gal infection seems less dependent on CAR than Ad $\beta$ gal.

\section{Discussion}

Gene therapy for cancer offers novel possibilities that will eventually be used in combination with existing therapies. However, data concerning the test of different viruses to transduce breast cancer cells are still limited. The goal of this study was to evaluate for the first time three types of vectors (Ad5, CAV-2 and AAV-2) in terms of their ability to transduce breast cancer cells.

We found that AAV-2 vectors were inefficient in transducing breast cancer cells. Although AAV-2 have shown great possibilities in restoring function of deficient genes in multiple organs (in particular in liver, muscle or brain) in mice (6), its efficacy in infection of tumor cells remains conflictual. Although Nguyen et al. (16) showed infection of human U87 glioblastoma cell line, CX1 metastatic colorectal carcinoma cells, and Hep3B hepatocellular carcinoma cells, successful delivery of AAV-2 to cancer cells remains rare. Our data are consistent with the idea that AAV-2 is not the ideal vector to transduce breast cancer cells.

We observed an excellent infection of most of the cell lines tested by Ad5 virus, whereas, CAV-2 vector showed a low infection ability. Human Ads transduce different types of tumors in colon (17), liver (18), ovary (19) or breast (20). CAV-2, similarly to Ad5 is thought to preferentially infect the upper respiratory tract, but previous reports have also shown that CAV-2 could infect a variety of neurons (21). Based on our data, it seems that CAV-2 vectors could be of interest in some limited cases. However, this disadvantage could be alleviated by the lower seroprevalence against CAV-2 in humans compared to Ad5, which could confer them a particular interest in breast cancer gene therapy. 
Concerning the mechanisms of cell transduction, CAR is thought to be the primary receptor for most human (22) and some nonhuman Ads (21). It is important to note that CAR expression has been shown to be decreased in bladder and renal cancers, whereas $\alpha_{v}$ integrin expression was enhanced $(23,24)$. On the other hand, CAR expression is enhanced in metastatic prostate cancers (25). The situation of breast cancers has not been studied systematically and needs to be evaluated. A recent study has also shown that CAR expression could be regulated by the Raf/MEK/ERK pathway in breast cancer cells (26), which suggests that multiple signals could regulate CAR levels. CAR-negative cells, which are transduced poorly by Ads, can be made susceptible to infection by transfection of CAR (27). However, we did not observe a significant correlation between CAR expression and Ad5 or CAV-2 infection, which is in good agreement with a previous study in ovarian cancers (28). Moreover, our blocking studies with CAR antibody showed that cell lines expressing comparable levels of CAR did not show the same sensitivity to the antibody. This further suggests that additional surface proteins are involved in adenovirus infection.

In particular, the internalization of Ads is thought to be facilitated by interaction between the arginine-glycine-aspartic acid (RGD) motif of the penton base, and integrins $\alpha \mathrm{v} \beta 3$ and $\alpha \mathrm{v} \beta 5$ (14). For these reasons, we asked whether these proteins could account for the differential infection by human and canine adenoviruses. According to our evaluation of integrin levels and to our blocking studies, integrins do not seem to play a major role in Ad5 or CAV-2 infection. For CAV-2 vectors, this could be explained because CAV-2 does not have an integrin interacting-motif (29) in the capsid, in contrast to most human Ads. Therefore, it seems reasonable to expect the interaction of the CAV-2 capsid with $\alpha \mathrm{v}$ integrins, to be less important. However, our study does not support a role for integrins in Ad5 infection. This is in agreement with previous studies showing that $\beta 5$ integrin $\mathrm{KO}$ mice are not less susceptible to Ad infection, which again suggests that integrins play a minor role in some tissues (30). On the other hand, Wickham and colleagues originally showed that CS-1 ( $\beta 5$-deficient) and M21 ( $\alpha$ v-deficient) cells became more susceptible to Ad infection when they were 
transfected with the complementary integrin dimer (14). But Soudais et al. (2001) (21) showed that in CS-1- $\beta 5$ and M21- $\alpha$ v cells, the level of CAR expression also increased and may have been responsible for the increase infection efficiency. In conclusion, if $\alpha v$ integrins contribute to the infection by Ad5 and CAV-2, there are not the primary determinants, which is in agreement with some studies performed in other types of cancer (19). Concerning AAV-2 vector tropism, heparan sulfate glycosaminoglycans (HSG) have been suspected to be the major receptor used by these viruses. However, competition studies with heparin that we performed on MDA-MB-436 and SKBR-3 cells did not show any involvement of HSG in viral transduction of these cells (data not shown). This suggests that other receptors might be involved in AAV-2 attachment to the cells.

In conclusion, our data suggest that Ad5 and to a lesser extent CAV-2 vectors could be of particular interest in targeting breast cancer cells. The development of new vectors with modified tropism will definitely improve the promising results obtained with these vectors and will help to propose alternative strategies to present anti-cancer therapies.

\section{ACKNOWLEDGEMENTS}

We are grateful to C. Duperray for his help in FACS studies. We thank the Vector Core of the University Hospital of Nantes supported by the Association Française contre les Myopathies (AFM) for the production of Adßgal and AAV $\beta$ gal. This work was supported by grants from ARC (Association pour la Recherche contre le Cancer, Grant No. 4302), la Ligue Nationale contre le Cancer (Comite du Gard), INSERM, and CNRS, Fondation pour La Recherche Medicale (EJK).

\section{REFERENCES}

1. Walt, A. J., Singhakowinta, A., Brooks, S. C., and Cortez, A. (1976) Surgery 80, 506-512. 
2. $\quad$ Cosman, F., and Lindsay, R. (1999) Endocr Rev 20, 418-434.

3. Vile, R. G., Russell, S. J., and Lemoine, N. R. (2000) Gene Ther 7, 2-8.

4. Mountain, A. (2000) Trends Biotechnol 18, 119-128.

5. Loser, P., Huser, A., Hillgenberg, M., Kumin, D., Both, G. W., and Hofmann, C. (2002) Curr Gene Ther 2 , 161-171.

6. $\quad$ Monahan, P. E., and Samulski, R. J. (2000) Gene Ther 7, 24-30.

7. Simon, W. E., Albrecht, M., Trams, G., Dietel, M., and Holzel, F. (1984) J Natl Cancer Inst 73, $313-321$.

8. $\quad$ Kremer, E. J., Boutin, S., Chillon, M., and Danos, O. (2000) J Virol 74, 505-512.

9. Grimm, D., Kern, A., Rittner, K., and Kleinschmidt, J. A. (1998) Hum Gene Ther 9, 2745-2760.

10. Lazennec, G., Bresson, D., Lucas, A., Chauveau, C., and Vignon, F. (2001) Endocrinology 142, 4120-4130.

11. Hemmi, S., Geertsen, R., Mezzacasa, A., Peter, I., and Dummer, R. (1998) Hum Gene Ther 9, $2363-2373$.

12. Berthet, V., Rigot, V., Champion, S., Secchi, J., Fouchier, F., Marvaldi, J., and Luis, J. (2000) J Biol Chem 275 , 33308-33313.

13. Hemminki, A., Kanerva, A., Kremer, E. J., Bauerschmitz, G. J., Smith, B. F., Liu, B., Wang, M., Desmond, R. A., Keriel, A., Barnett, B., Baker, H. J., Siegal, G. P., and Curiel, D. T. (2003) Mol Ther 7, 163-173.

14. Wickham, T. J., Mathias, P., Cheresh, D. A., and Nemerow, G. R. (1993) Cell 73, 309-319.

15. Summerford, C., Bartlett, J. S., and Samulski, R. J. (1999) Nat Med 5, 78-82.

16. Nguyen, J. T., Wu, P., Clouse, M. E., Hlatky, L., and Terwilliger, E. F. (1998) Cancer Res 58, 5673-5677.

17. Hirschowitz, E. A., Ohwada, A., Pascal, W. R., Russi, T. J., and Crystal, R. G. (1995) Hum Gene Ther 6, 1055-1063.

18. Kaneko, S., Hallenbeck, P., Kotani, T., Nakabayashi, H., McGarrity, G., Tamaoki, T., Anderson, W. F., and Chiang, Y. L. (1995) Cancer Res 55, 5283-5287.

19. You, Z., Fischer, D. C., Tong, X., Hasenburg, A., Aguilar-Cordova, E., and Kieback, D. G. (2001) Cancer Gene Ther 8, 168-175.

20. Seth, P., Brinkmann, U., Schwartz, G. N., Katayose, D., Gress, R., Pastan, I., and Cowan, K. (1996) Cancer Res. 56, 1346-1351.

21. Soudais, C., Laplace-Builhe, C., Kissa, K., and Kremer, E. J. (2001) Faseb J 15, 2283-2285.

22. Roelvink, P. W., Lizonova, A., Lee, J. G., Li, Y., Bergelson, J. M., Finberg, R. W., Brough, D. E., Kovesdi, I., and Wickham, T. J. (1998) J Virol 72, 7909-7915.

23. Sachs, M. D., Rauen, K. A., Ramamurthy, M., Dodson, J. L., De Marzo, A. M., Putzi, M. J., Schoenberg, M. P., and Rodriguez, R. (2002) Urology 60, 531-536.

24. Okegawa, T., Pong, R.-C., Li, Y., Bergelson, J. M., Sagalowsky, A. I., and Hsieh, J.-T. (2001) Cancer Res 61, 6592-6330.

25. Rauen, K. A., Sudilovsky, D., Le, J. L., Chew, K. L., Hann, B., Weinberg, V., Schmitt, L. D., and McCormick, F. (2002) Cancer Res 62, 3812-3818.

26. Anders, M., Christian, C., McMahon, M., McCormick, F., and Korn, W. M. (2003) Cancer Res 63, $2088-2095$.

27. Bergelson, J. M., Cunningham, J. A., Droguett, G., Kurt-Jones, E. A., Krithivas, A., Hong, J. S., Horwitz, M. S., Crowell, R. L., and Finberg, R. W. (1997) Science 275, 1320-1323.

28. Bruning, A., Kohler, T., Quist, S., Wang-Gohrke, S., Moebus, V. J., Kreienberg, R., and Runnebaum, I. B. (2001) Hum Gene Ther 12, 391-399.

29. Sonnenberg, A. (1993) Curr Top Microbiol Immunol 184, 7-35.

30. Huang, X., Griffiths, M., Wu, J., Farese, R. V., Jr., and Sheppard, D. (2000) Mol Cell Biol 20, 755-759. 


\section{TABLES}

Table 1. Maximal pourcentage of infected cells by Ad $\beta$ gal, CAV $\beta$ gal or AAV $\beta$ gal

\begin{tabular}{|c|c|c|c|}
\hline & Ad $\beta$ gal & CAV $\beta$ gal & AAV $\beta$ gal \\
\hline MDA-MB-231 & $91 \pm 7.2$ & $10 \pm 1.8$ & $0.1 \pm 0.01$ \\
\hline MDA-MB-435 & $15 \pm 1.9$ & $5.0 \pm 1.2$ & $1.0 \pm 0.15$ \\
\hline MDA-MB-436 & $100 \pm 1.1$ & $70 \pm 3.2$ & $85 \pm 4.6$ \\
\hline SKBR-3 & $100 \pm 0.9$ & $50 \pm 0.8$ & $55 \pm 1.1$ \\
\hline EFM-19 & $0.9 \pm 0.2$ & $1.0 \pm 0.2$ & $2.0 \pm 1.4$ \\
\hline MCF-7 & $64 \pm 5.3$ & $40 \pm 2.8$ & $5 \pm 0.43$ \\
\hline T47-D & $81 \pm 9.6$ & $50 \pm 1.2$ & $1.0 \pm 0.16$ \\
\hline ZR-75-1 & $33 \pm 4.3$ & $0.2 \pm 0.02$ & 0 \\
\hline
\end{tabular}

Maximal infection efficiencies with Ad $\beta$ gal, CAV $\beta$ gal and AAV $\beta$ gal vectors. Breast cancer cells were infected at increasing MOIs ( 0 to 75 for Ad $\beta$ gal and CAV $\beta$ gal / 0 to 35 for AAV $\beta g a l$ ) and the maximal pourcentage \pm SD of infected cells was determined for each cell line. 


\section{FIGURE LEGENDS}

Fig. 1. Infectivity of breast cancer cell lines by Ad $\beta$ gal virus. A. ER-negative (MDA-MB-231, MDA-MB-435, MDA-MB-436, SKBR-3) or Estrogen-Receptor-positive (EFM-19, MCF-7, T47-D, ZR-75-1) breast cancer cells were infected with Ad $\beta$ gal virus at increasing multiplicity of infection (MOI) $(0,1,5,2575,375)$. The infected cells were then grown for $48 \mathrm{~h}$ and stained for $\beta$ gal activity. B. Quantification of $\beta$-galactosidase positive cells after Ad infection at increasing MOI (0 to 375). $\beta$-galactosidase-positive cells were counted from 3 distinct experiments ( $\mathrm{SD}<15 \%)$. Be aware of the change of scale for MOI > 100.

Fig. 2. CAR and $\alpha v$ integrin levels in breast cancer cell lines. A. CAR and $\alpha v$ integrin RNA levels were monitored using quantitative PCR. Results are expressed as arbitrary units corresponding to the ratio of CAR or $\alpha_{v}$ levels normalized by rS9 levels and represent the mean \pm SD of three independent experiments. B. CAR, $\alpha_{v} \beta 5$ and total $\alpha_{v}$ protein expression was monitored by flow cytometry. Here is represented an example of data obtained with MDA-MB-231 cells. The dotted line corresponds to the negative control (only secondary antibody), whereas the grey filled curve corresponds to the staining with the specific antibody. C. The relative expression of the above markers was expressed as the relative mean fluorescence (ratio specific/negative control). Results represent the mean of 3 independent experiments \pm SD.

Fig. 3. Analysis of CAR and integrins involvement in viral infection. MDA-MB-436, MCF-7 and T47-D cells were incubated with whole rabbit serum (mock), $\alpha \mathrm{V}(\alpha \mathrm{V}$ Ab) or CAR (CAR Ab) antibodies prior to infection with Ad $\beta$ gal or CAV $\beta$ gal viruses at MOI 75. Results are expressed as \% of 
Lucas et al.

mock treated cells infected with Ad $\beta$ gal or CAV $\beta$ gal viruses and represent the mean of 3 independent experiments \pm SD. 